Colorectal cancer

\section{Obesity, gender, and colon cancer}

\section{E Giovannucci}

\section{Obesity is associated with an increased risk of colorectal cancer in premenopausal but not postmenopausal women}

M any studies have shown that a high body mass index (BMI) is related to an increased risk of colon cancer in men but this relationship has not been as consistent in studies of women. However, an association between BMI and colon cancer risk is observed in cohorts of relatively young women, and among younger women in case control studies that stratified results by age. ${ }^{1}$ In addition, a recent cohort study found a positive association between BMI and colorectal cancer among women less than 55 years of age but not among older women. ${ }^{2}$ These studies did not examine the association between BMI and colon cancer risk by menopausal status but the clearer association with younger women suggested that an association between BMI and colon cancer risk may be stronger for, or perhaps even limited to, premenopausal women.

A study by Terry and colleagues ${ }^{3}$ in the current issue of Gut tested the hypothesis that menopausal status modifies the relationship between BMI and colon cancer risk [see page 191]. The investigators did indeed find a strong association between BMI and colon cancer risk in premenopausal but not postmenopausal women. The test for interaction was statistically significant $(\mathrm{p}=0.01)$. As seen in most other studies, ${ }^{1}$ BMI appeared to be associated with a risk of colon cancer but not rectal cancer. The data confirm that obesity is a risk factor for colon cancer but suggest its influence diminishes in aging women, probably related to menopausal status.

Two obvious questions arise: why does obesity increase the risk of colon cancer, and why does being postmenopausal weaken this effect? We do not know the answers definitively but obesity and menopausal status are strong determinants or indicators of concentrations for various hormones that may be critical for colon carcinogenesis. At least three hormonal axes, insulin-like growth factors (IGFs), insulin, and oestrogen, may be relevant. Hyperinsulinaemia is strongly related to physical inactivity, high body mass, and central deposition of adipose tissue, all risk factors for colon cancer. Recently, insulin has been shown to be a colon tumour promoter in an animal model, and hyperinsulinaemia and adult onset diabetes mellitus are related to the risk of colon cancer. ${ }^{1}$ A cancer enhancing effect of insulin is likely to act through a mediating factor because supraphysiological levels of insulin are required for its mitogenic effects in vitro. A strong candidate for a mechanism is the role of insulin in lowering IGF binding protein 1 (IGFBP-1) levels, which thereby increase free IGF-1 levels. ${ }^{4}$

Oestrogens also appear to influence colorectal cancer risk. Although studies on reproductive factors and colorectal cancer have provided inconsistent results, postmenopausal hormone use has been associated with a decreased risk of colon or colorectal cancer with remarkable consistency. ${ }^{6}$ However, these results are from observational studies and need to be confirmed in randomised intervention trials. While exogenous oestrogens may confer some protection against colorectal cancer, as suggested by the epidemiological data, the role of endogenous oestrogens on colorectal cancer risk has not been adequately studied. In premenopausal women, ovarian sources of oestrogen are of prime importance but in postmenopausal women, conversion of androgens to oestrogen in adipose tissue is the major source of oestrogens. Thus BMI is correlated with oestrogen levels in postmenopausal women.

A working hypothesis to explain the observations concerning BMI and colon cancer risk is that obesity increases risk through the insulin/IGF axis; however, higher concentrations of oestrogens associated with obesity in postmenopausal women may have a countering influence. ${ }^{2}$ The opposing influences of the insulin and IGF axis and oestrogen appear to approximately cancel out each other, leading to no appreciable association between BMI and colorectal cancer risk in postmenopausal women. In premenopausal women, obesity increases insulin concentrations but is a relatively unimportant source of oestrogens because ovarian production is high. Thus in premenopausal women, the adverse influence of obesity on hyperinsulinaemia predominates.

This hypothesis is far from proved but it does provide a focus for future studies.
The role of insulin and IGF is supported by a large body of data, reviewed in detail elsewhere. ${ }^{1}$ If we assume this aspect of the hypothesis to be correct, we need to account for the apparent weak or null effect of obesity, a strong determinant of hyperinsulinaemia, on colon cancer risk in older women. The finding by Terry et al that menopausal status modifies the relationship between BMI and colon cancer needs to be confirmed in other studies. ${ }^{3}$ A number of existing cohort and case control studies can readily address this relationship. It is also crucial to examine the influence of endogenous oestrogens in relation to colorectal cancer risk, preferably in prospectively collected blood samples among women not using exogenous oestrogens. In such a study, insulin and IGF-1 should also be measured so that the influences of hormones independent of each other, and of obesity, can be assessed. If this hypothesis is correct, a further prediction is that obesity should remain a significant risk factor in postmenopausal women taking replacement oestrogens because the oestrogenic effect of higher BMI would be relatively less important for these women.

The provocative findings by Terry et al should provide a stimulus for further work. Future studies in this area may be able to provide a more complete understanding of the causes of colon cancer. None the less, although we do not have a complete understanding of the mechanisms, these findings further underscore the importance of weight control for women as well as men.

Gut 2002;51:147

\section{Author's affiliation}

E Giovannucci, Harvard Medical School, Channing Laboratory, 181 Longwood Avenue, Boston, MA 02115, USA;

edward.giovannucci@channing.havard.edu

\section{REFERENCES}

1 Giovannucci E. Insulin, insulin-like growth factors and colon cancer: a review of the evidence. J Nutr 2001;131:3109-20S

2 Terry P, Giovannucci E, Bergkvist L, et al. Body weight and colorectal cancer risk in a cohort of Swedish women: relation varies by age and cancer site. $\mathrm{Br} J$ Cancer 2001;85:346-9.

3 Terry PD, Miller AB, Rohan TE. Obesity and colorectal cancer risk in women. Gut 2002:51:191-4

4 Powell DR, Suwanichkul A, Cubbage ML, et al. Insulin inhibits transcription of the human gene for insulin-like growth factor-binding protein-1. J Biol Chem 1991;266:18868-76.

5 Lee PD, Conover CA, Powell DR. Regulation and function of insulin-like growth factor-binding protein-1. Proc Soc Exp Biol Med 1993;204:4-29

6 La Vecchia C, Brinton LA, McTiernan A. Menopause, hormone replacement therapy and cancer. Maturitas 2001:39:87-1 15.

7 Bulun SE, Zeitoun K, Sasano H, et al. Aromatase in aging women. Semin Reprod Endocrinol 1999;17:349-58. 